\title{
Tetrandrine suppresses cervical cancer growth by inducing apoptosis in vitro and in vivo
}

This article was published in the following Dove Press journal:

Drug Design, Development and Therapy

\author{
Haiyan Zhang ${ }^{1,2}$ \\ Beibei $\mathrm{Xie}^{2}$ \\ Zhen Zhang² \\ Xiugui Sheng ${ }^{3}$ \\ Shiqian Zhang' \\ 'Department of Gynecology, \\ Affiliated Qilu Hospital of Shandong \\ University, Linyi, People's Republic of \\ China; ${ }^{2}$ Department of Gynecology \\ Ward-I, Linyi City People's Hospital, \\ Linyi, People's Republic of China; \\ ${ }^{3}$ Department of Gynecology, Cancer \\ Hospital Chinese Academy of Medical \\ Sciences, Beijing, People's Republic \\ of China
}

\begin{abstract}
Introduction and aim: Cervical cancers are the most common forms of cancer that occur in women globally and are difficult to be cured in their terminal stages. Tetrandrine (TET), a monomeric compound isolated from a traditional Chinese medicine, Radix Stephania tetrandrae, exhibits anticancer effects on different tumor types. However, the mechanisms by which TET regulates the proliferation, apoptosis, migration, and invasion in cervical cancer remain unclear. Thus, this study aimed to investigate the therapeutic effects of TET on cervical cancer in vitro and in vivo.
\end{abstract}

Methods: Cell Counting Kit-8, immunofluorescence, flow cytometry, wound healing, and transwell migration assays were used to detect cell proliferation, apoptosis, and migration and invasion, respectively, in vitro. In addition, immunohistochemical assays were performed to evaluate tumor growth and apoptosis in vivo. Moreover, Western blotting was used to examine active caspase 3, matrix metalloproteinase (MMP)2, and MMP9 protein levels in vitro and in vivo.

Results: The results revealed that TET significantly inhibited SiHa cell proliferation in vitro and suppressed tumor growth in vivo. Meanwhile, TET was revealed to induce cervical cancer cell apoptosis by upregulating active caspase 3 in vitro and in vivo. Furthermore, the migration and invasion of SiHa cells were inhibited by TET accompanied with MMP2 and MMP9 downregulation.

Conclusion: We have shown that TET inhibited cervical tumor growth and migration in vitro and in vivo for the first time. The accumulating evidence suggests that TET could be a potential therapeutic agent for the treatment of cervical cancer.

Keywords: tetrandrine, cervical cancer, proliferation, apoptosis, migration

\section{Introduction}

Cervical cancer is the second most common malignancy among women worldwide. ${ }^{1}$ Every year, more than 500,000 new cases of cervical cancer are diagnosed. ${ }^{2}$ Human papillomavirus (HPVs) is considered to be a primary pathogenic factor for cervical cancer. ${ }^{3}$ More than 100 HPV types have been identified, including high-risk HPV and low-risk HPV based on oncogenic potential. ${ }^{4}$ The current therapeutic treatments for cervical cancer mainly consist of surgery, radiation, and chemotherapy. ${ }^{5}$ Although radiotherapy has high definitive cure rates in patients with early stage disease, the cure rates in patients with advanced cervical cancer remain poor. ${ }^{6}$ The 5 -year overall survival rate is only $66 \%$ in patients with cervical cancer. ${ }^{6}$ Therefore, more effective therapeutic strategies for treating cervical cancer are to be discovered.

Tetrandrine (TET), a bisbenzylisoquinoline alkaloid, is isolated from the root tuber of Radix Stephania tetrandrae S. ${ }^{7}$ TET has been used as an effective monomeric compound to remedy patients with inflammation, hypertension, arrhythmia, and arthritis. ${ }^{8}$ In addition, TET has been demonstrated to mediate neuroinflammation
Correspondence: Shiqian Zhang Department of Gynecology, Affiliated Qilu Hospital of Shandong University, No 27, Jiefang East Road, Lanshan District, Linyi, Shandong 276000, People's Republic of China Email shiqian_zhang99@126.com 
in Alzheimer's disease by decreasing the expression of proinflammatory mediators by inhibition of NF- $\kappa$ B activation. ${ }^{9}$ TET also significantly reduced the intraocular pressure levels in the hypertensive eyes in animal model with ocular hypertension. ${ }^{10}$

Several studies have shown that TET exhibited strong antitumor actions on gastric cancer, hepatocellular carcinoma, lung cancer, gliomas, and colon cancer in vitro and in vivo. ${ }^{11-15} \mathrm{Li}$ et al reported that TET exerted antitumor activity on gastric cancer via activation of the apoptosis mediator caspase $3 .{ }^{11}$ In spite of many reports about the anticancer activities of TET, little is known about the effect of TET on human cervical cancer. And the mechanisms underlying the antitumor effect of TET on cervical cancer have not yet been illuminated. Hence, this study aimed to investigate the effects and mechanisms of TET on the cervical cancer in vitro and in vivo.

\section{Materials and methods Cell line and cell culture}

The human cervical cancer cell line $\mathrm{SiHa}$ was purchased from American Type Culture Collection (ATCC, Rockville, MD, USA). The cells were maintained in Dulbecco's Modified Eagle Medium (DMEM) supplemented with 10\% fetal bovine serum (FBS) (Gibco, Carlsbad, CA, USA) and 1\% penicillin and streptomycin (100 units/mL). The cells were incubated at $37^{\circ} \mathrm{C}$ in a humidified atmosphere of $5 \% \mathrm{CO}_{2}$ in air.

\section{Cell Counting Kit-8 (CCK-8) assay was used to determine cell viability}

TET was dissolved in dimethyl sulfoxide (DMSO) and diluted in culture medium immediately before use. Cell viability was determined using $\mathrm{CCK}-8$ assay (Beyotime Institute of Biotechnology, Haimen, China) according to the manufacturer's protocols. SiHa cells were seeded into a 96-well plate at a density of $5 \times 10^{3}$ cells/well and then treated with different concentration of TET $(0,1,3,10$, or $30 \mu \mathrm{M}$ ) for 24,48 , and 72 hours. After incubation, $10 \mu \mathrm{L}$ CCK-8 reagent was added to each well and then incubated for 2 hours. The absorbance values were measured at $450 \mathrm{~nm}$ wavelength (Bio-Rad Laboratories, Benicia, CA, USA). TET (purity $>95 \%$ ) standard product was purchased from Sigma (Sigma-Aldrich, St Louis, MO, USA).

\section{Flow cytometric analysis of cell apoptosis}

SiHa cells were incubated in six-well plates at a density of $4 \times 10^{5}$ cells/well overnight, and then treated with 3 or $10 \mu \mathrm{M}$ TET for another 48 hours. The cells were treated with $0.2 \%$
DMSO (Sigma-Aldrich) as control. Afterward, the cell suspension was mixed with $10 \mu \mathrm{L}$ Annexin $\mathrm{V}$ and $5 \mu \mathrm{L}$ propidium iodide (PI) for 10 minutes at room temperature in the dark according to the manufacturer's protocols (Thermo Fisher Scientific, Waltham, MA, USA). Cell apoptosis was analyzed using a fluorescence activated cell sorting (FACS) flow cytometer (BD Bioscience, San Jose, CA, USA).

\section{Immunofluorescence}

SiHa cells were exposed to DMSO, 3 or $10 \mu \mathrm{M}$ TET at $37^{\circ} \mathrm{C}$ for 48 hours. Then, the incubated cells were washed three times with $\mathrm{PBS}$, prefixed in $4 \%$ paraformaldehyde for 10 minutes at room temperature, and then fixed in pre-cold methanol for 10 minutes at $-20^{\circ} \mathrm{C}$. Later on, cells were incubated with primary antibodies for anti-Ki67 (Abcam, Cambridge, UK) $(1: 1,000)$, DAPI $(1: 1,000)$ at $4^{\circ} \mathrm{C}$ overnight. Subsequently, cells were incubated with secondary antibodies (Abcam) $(1: 2,000)$ at $37^{\circ} \mathrm{C}$ for 1 hour. The samples were observed by fluorescence microscope at once (Olympus, Tokyo, Japan).

\section{Western blot analysis}

SiHa cells were incubated in six-well plates at a density of $4 \times 10^{5}$ cells/well overnight and then treated with different concentration of TET $(0,3$, or $10 \mu \mathrm{M})$ for 48 hours at $37^{\circ} \mathrm{C}$ in $\mathrm{CO}_{2}$ incubator. Later on, cultured cells were lysed using RIPA lysis buffer and Bradford Protein Assay Kit (Beyotime Institute of Biotechnology) was used to quantify the protein concentration. Equal amounts of proteins $(50 \mu \mathrm{g})$ were separated by sodium dodecyl sulfate-polyacrylamide gel electrophoresis (SDS-PAGE) with 10\% SDS polyacrylamide gel, and the proteins were then transported to polyvinylidene fluoride membranes (PVDF; Thermo Fisher Scientific) in 2 hours. The membranes were blocked with 5\% skimmed milk in Tris-Buffered Saline with Tween 20 (TBST) for 1 hour at room temperature. Then, the membranes were washed three times in TBST and incubated with primary antibodies: antiactive caspase 3 (Abcam), anti-matrix metalloproteinase (MMP)2 (Abcam), anti-MMP2 (Abcam), and anti- $\beta$-actin (Abcam). After that, the PVDF membrane was incubated with anti-rabbit (Abcam) $(1: 2,000)$ secondary antibody at room temperature for 1 hour before being determined by chemiluminescence. Finally, the PVDF membranes were incubated with ECL reagent (Santa Cruz Biotechnology) to detect the blots. The densitometric values of the bands were measured using Image Quant TL software (v 8.1; GE Life Sciences, Chicago, IL, USA). The density of blots for targets was normalized to $\beta$-actin. 


\section{Matrigel invasion assay}

The cell invasiveness assay was performed using a 24-well matrigel chambers according to manufacturer's protocol (BD, Franklin Lake, NJ, USA). Transwell membranes (polycarbonic membrane, diameter $6.5 \mathrm{~mm}$, pore size $8 \mu \mathrm{m}$ ) were coated with matrigel in a total of $50 \mu \mathrm{L}$ of each transwell (matrigel/serum free medium: 1/5). Briefly, SiHa cells $\left(4 \times 10^{5}\right.$ cells/well $)$ with TET $(0,3$, or $10 \mu \mathrm{M})$ were seeded onto the upper chamber in serum-free medium, and the bottom wells in the system were added with DMEM medium containing $10 \% \mathrm{FBS}$. After incubation for 72 hours at $37^{\circ} \mathrm{C}$, cells on the upper surface were scraped and washed away by a cotton swab, whereas the invaded cells on the underside were fixed with $4 \%$ formaldehyde and stained with $0.05 \%$ crystal violet for 2 hours. Finally, invaded cells were pictured and counted under a microscope.

\section{Wound healing assay}

$\mathrm{SiHa}$ cells were seeded into six-well plate at a density of $4 \times 10^{5}$ cells/well. After 24 hours of incubation, a "scratch" was made in the cell monolayer to create a wound, and the cells were treated with different concentration of TET $(0,10 \mu \mathrm{M})$ for 48 hours at $37^{\circ} \mathrm{C}$ in $\mathrm{CO}_{2}$ incubator. Wound healing was observed and pictured at 0 and 48 hours under inverted microscope at $100 \times$ magnification (Leica, Buffalo Grove, IL, USA). Wound healing was analyzed using ImageJ software (National Institutes of Health).

\section{Animal study}

To investigate the effects of TET on the cervical tumorburdened animals, 12 female BALB/nude mice (aged 4-6 weeks) were purchased from Shanghai Slac Animal Center (Shanghai, China) and housed within a dedicated SPF facility with alternating 12 hours periods of light and darkness, a constant temperature of $18^{\circ} \mathrm{C}-23^{\circ} \mathrm{C}$, and $55 \%-65 \%$ humidity. Aliquots of SiHa cells $\left(5 \times 10^{6}\right.$ cells in $100 \mu \mathrm{L}$ of PBS) were injected subcutaneously into the right inguinal area of the mice. The tumor growth and body weight of the mice were monitored weekly. When the volume of the tumor reached $180 \mathrm{~mm}^{3}$, tumor-bearing mice were randomly divided into treatment and vehicle groups $(n=4)$. TET was administered via oral gavage at doses of 20 or $50 \mathrm{mg} / \mathrm{kg} / \mathrm{day}$. The vehicle group received normal saline only. Tumor volume was measured by calipers weekly until mice were sacrificed under anesthesia. Each tumor was excised and weighed when nude mice were sacrificed on day 29. Parts of each tumor tissue were wax embedded for immunohistochemical staining in vivo. All animal experiments were performed in accordance with the National Institutes of Health guide for the care and use of laboratory animals, following a protocol approved by the Ethics Committees of Affiliated Qilu Hospital of Shandong University.

\section{Immunohistochemical analyses}

Ki67-positive cells were determined by immunohistochemical staining. Tumor sample preparation and immunohistochemical staining were performed according to a previous study. ${ }^{16}$ Briefly, the tissue sections were pretreated with Tris-EDTA buffer solution $\left(\mathrm{pH} \mathrm{9.0)}\right.$ at $95^{\circ} \mathrm{C}$. After incubation with the primary antibody for 60 minutes, a biotinylated immunoglobulin cocktail of goat anti-rabbit IgG was applied for 30 minutes at room temperature. Visualization was performed using a polymer immunohistochemical detection system (EnVision kit; Dako Japan). The rate of Ki67-positive cell was determined by counting in each sample.

\section{TUNEL staining}

Deparaffinized tissue sections were stained using an APOBrdUTM TUNEL Assay Kit (Thermo Fisher Scientific) according to the manufacturer's instructions.

\section{Statistical analysis}

Data are means \pm SD and were obtained from at least three independent experiments. The comparison between two groups was analyzed by Student's $t$-test. The comparisons among multiple groups were made with one-way ANOVA followed by Dunnett's test. $P<0.05$ or $P<0.01$ was considered to indicate a statistically significant difference $\left(* P<0.05,{ }^{*} * P<0.01\right)$.

\section{Results}

\section{TET suppressed the proliferation of $\mathrm{SiHa}$ cells}

The chemical structure of TET is shown in Figure 1A. CCK-8 assay was used to determine the effects of TET on the viability of SiHa. As presented in Figure 1B, TET suppressed the proliferation of $\mathrm{SiHa}$ in a dose- and time-dependent manners. Therefore, the number of cells significantly decreased by $>50 \%$ following treatment with $30 \mu \mathrm{M}$ TET. TET at doses of 3 and $10 \mu \mathrm{M}$ was used in the following in vitro experiments. The results suggested that TET significantly inhibited the proliferation of $\mathrm{SiHa}$ cells in vitro.

\section{TET induced apoptosis in SiHa cells}

Since a significant inhibitory effect of TET on the proliferation of SiHa cells was observed, whether TET could 
A<smiles></smiles>

B

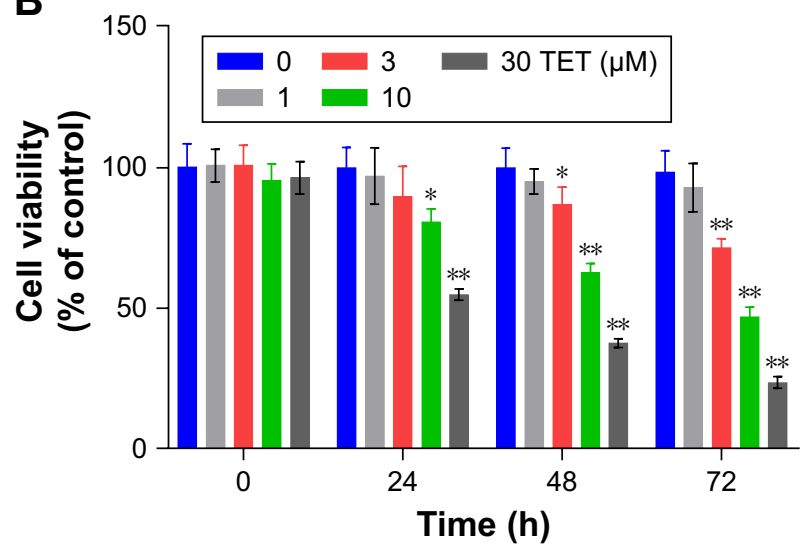

Figure I TET suppressed SiHa cells proliferation.

Notes: (A) The chemical structure of TET. (B) The viability of SiHa cells treated with TET $(0,1,3,10$, or $30 \mu M)$ for 24,48 , and 72 hours was investigated with CCK-8 assays. $* P<0.05, * * p<0.01$ vs DMSO group, $n=3$.

Abbreviations: CCK-8, Cell Counting Kit-8; DMSO, dimethyl sulfoxide; TET, tetrandrine.

induce apoptosis in $\mathrm{SiHa}$ cells was evaluated by Annexin V/PI staining. As indicated in Figure 2A and B, the apoptotic rates in cells treated with 3 or $10 \mu \mathrm{M}$ TET were $\sim 10 \%$ and $30 \%$, respectively. Ki67 was used as a nuclear marker to identify proliferating cell. Immunofluorescence assays were performed to further investigate the effect of TET on proliferating cell. As presented in Figure 2C and D, both 3 and $10 \mu \mathrm{M}$ TET exhibited markedly anti-proliferating effects on SiHa cells compared with the DMSO group $(P<0.05$, $P<0.01$, respectively).

Next, the level of apoptosis-associated protein active caspase 3 was detected with Western blotting. The results showed that the expression of active caspase 3 was markedly increased in TET-treated cells compared with the DMSO group $(P<0.01)$ (Figure $2 \mathrm{E}$ and F). All these data suggested that TET inhibited the proliferation of SiHa cells by inducing apoptosis in vitro.

A
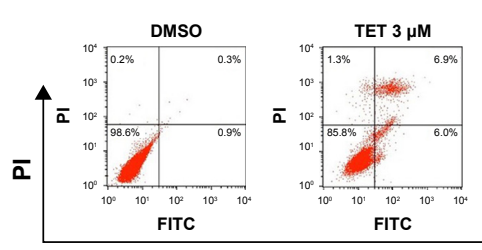

Annexin V

D

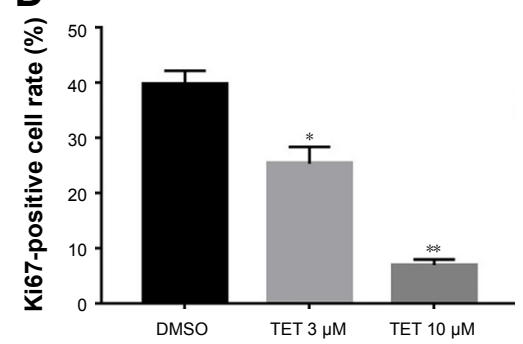

B
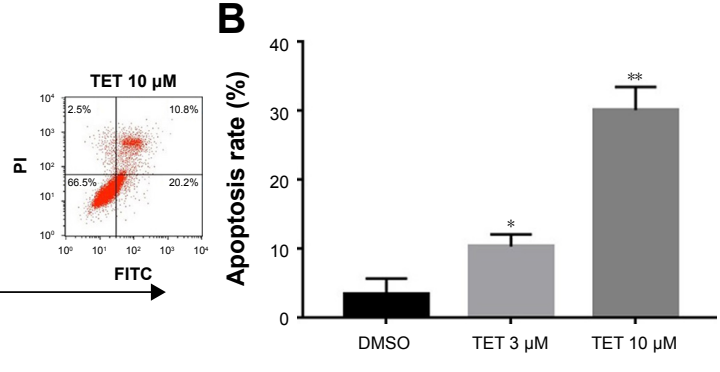

C ${ }_{\text {кi67 }}$

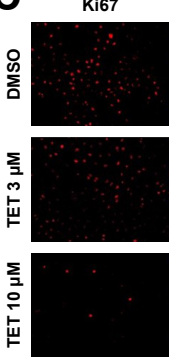

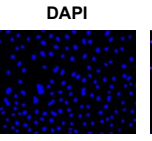
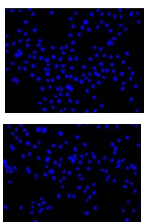

$\mathbf{F}$

E

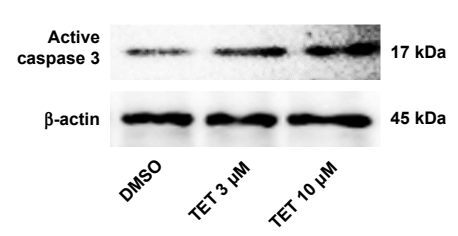

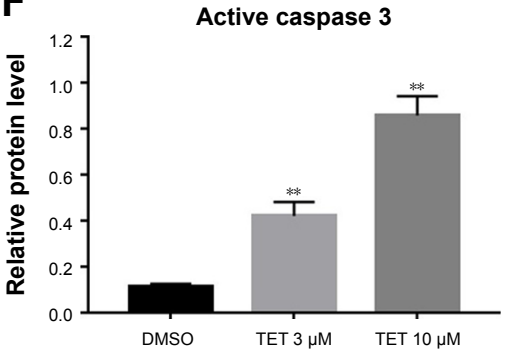

Figure 2 TET induced SiHa cells apoptosis.

Notes: (A) SiHa cells were treated with TET (0, 3, or $10 \mu \mathrm{M})$ for 48 hours and apoptotic cells were detected with Annexin V/PI double staining assay. (B) Apoptotic cell rates were calculated in each group. $* P<0.05, * * P<0.01$ vs DMSO group, $n=3$. (C) SiHa cells were treated with TET $(0,3$, or $10 \mu M)$ for 48 hours, and Ki67-positive cells were detected with immunofluorescence assay. (D) Relative Ki67-positive cell rates were quantified in each group. $* P<0.05, * * P<0.0$ I vs DMSO group, $n=3$. (E) Expressions of active caspase 3 in SiHa cells treated with TET $(0,3$, or $10 \mu \mathrm{M})$ for 48 hours were investigated with Western blotting. $\beta$-actin was used as an internal control. (F) The relative expressions of active caspase 3 were quantified by normalizing to $\beta$-actin in each group. ${ }^{*} * \mathrm{p}<0.01$ vs DMSO group, $\mathrm{n}=3$.

Abbreviations: DMSO, dimethyl sulfoxide; PI, propidium iodide; TET, tetrandrine. 


\section{TET inhibited SiHa cells migration and invasion}

Next, transwell assay and wound healing assay were performed to investigate the effects of TET on migration and invasion of SiHa cells. The data revealed that $10 \mu \mathrm{M}$ TET significantly attenuated the invasion capacity of SiHa cells compared with the DMSO group $(P<0.01)$ (Figure 3A and B). Consistent with this finding, wound healing assay showed that $10 \mu \mathrm{M}$ TET effectively suppressed the migration of $\mathrm{SiHa}$ cells as well (Figure $3 \mathrm{C}$ and D). In addition, cancer cell migration and invasion-associated proteins MMP2 and MMP9 were detected by Western blotting. As revealed in Figure 3E-G, levels of MMP2 and MMP9 were markedly downregulated in TET-treated SiHa cells compared with the DMSO group $(P<0.01)$. These data suggested that TET reduced the migration and invasion of $\mathrm{SiHa}$ cells by inhibiting the expressions of MMP2 and MMP9.

\section{TET inhibited cervical tumor growth by inducing apoptosis in vivo}

To further evaluate the antitumor effect of TET on cervical cancer in vivo, SiHa tumor bearing xenograft was applied. As shown in Figure 4A-C, both 20 and $50 \mathrm{mg} / \mathrm{kg}$ TET markedly inhibited tumor growth compared with vehicle group. In addition, the result of immunohistochemistry revealed that Ki67-positive cells were significantly decreased by $50 \mathrm{mg} / \mathrm{kg}$
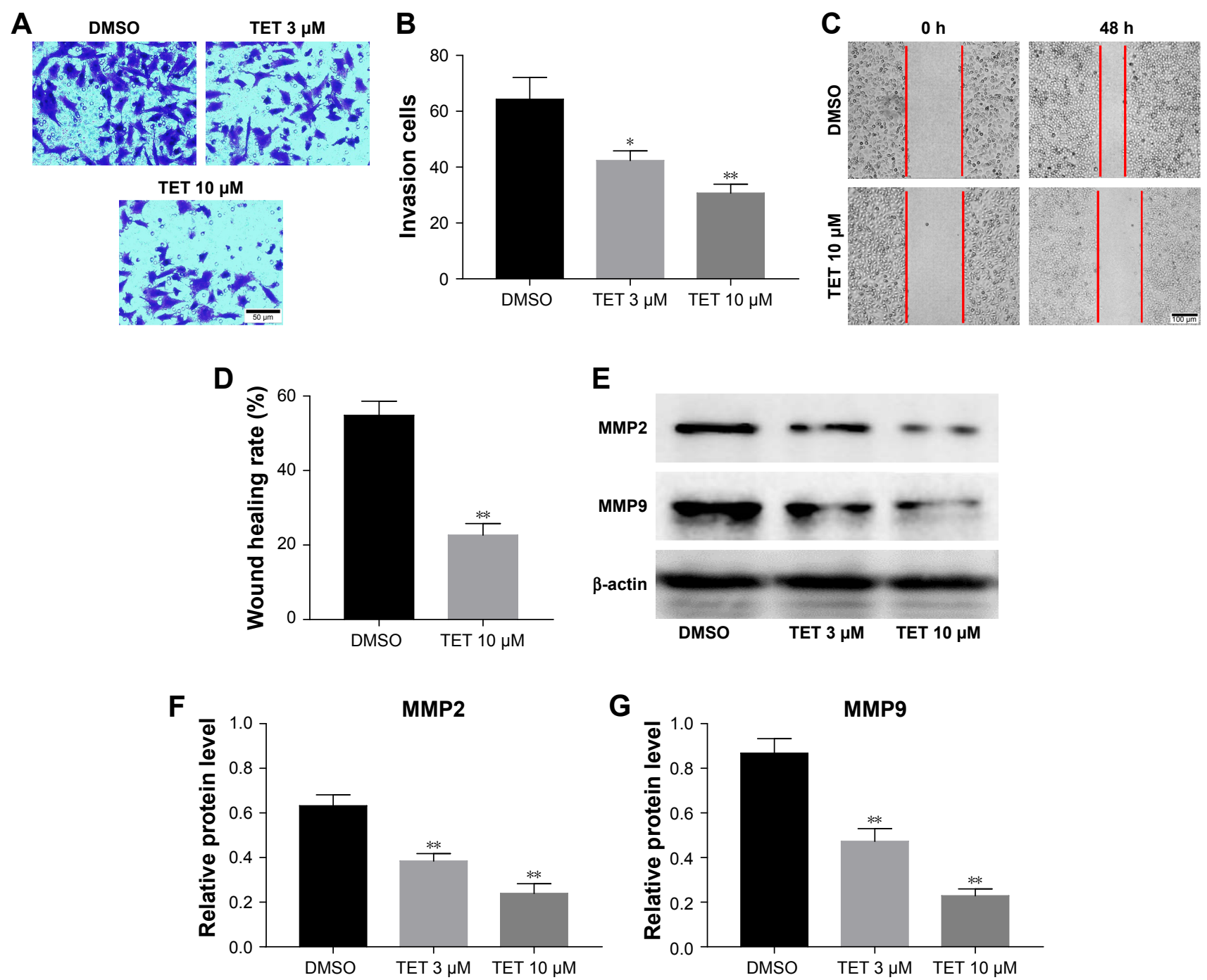

Figure 3 TET inhibited the migration and invasion of $\mathrm{SiHa}$ cells.

Notes: Cell invasion (A) and invasion cells (B) of SiHa cells following treatment with TET (0, 3 or I0 $\mu \mathrm{M})$ for $48 \mathrm{~h}$. $* P<0.05$, $* * P<0.0 \mathrm{I}$ vs DMSO group. Wound healing assay (C) and wound healing rate (D) of SiHa cells following treatment with TET (0 or $10 \mu \mathrm{M})$ for $48 \mathrm{~h}$. **P $<0.0$ I vs DMSO group. (E) Expression levels of MMP2 and MMP9 in $\mathrm{SiHa}$ cells treated with TET for 48 hours were detected with Western blotting. $\beta$-actin was used as an internal control. (F) The relative expression of MMP2 was quantified by normalizing to $\beta$-actin. $* * P<0.0$ I vs DMSO group, $n=3$. (G) The relative expression of MMP9 was quantified by normalizing to $\beta$-actin. $* * P<0.0$ I vs $D M S O$ group, $n=3$. Abbreviations: DMSO, dimethyl sulfoxide; MMP, matrix metalloproteinases; TET, tetrandrine. 
A

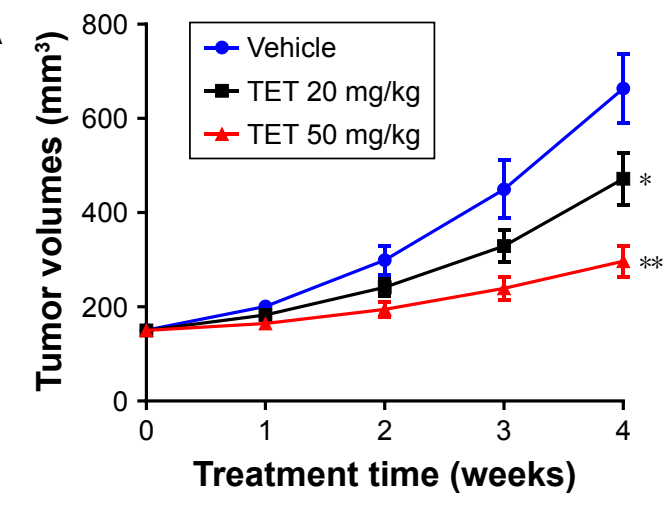

B

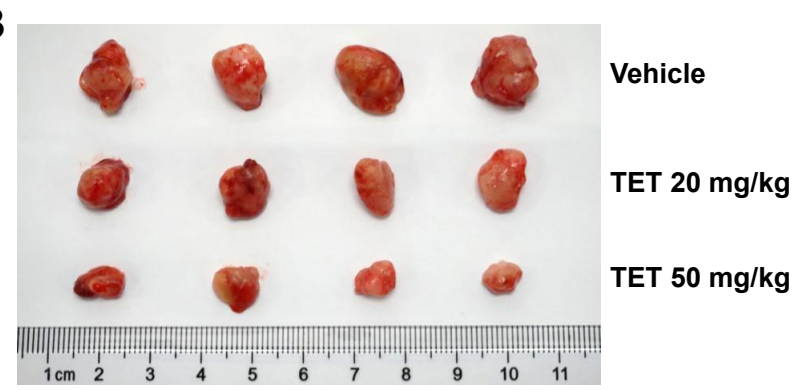

C

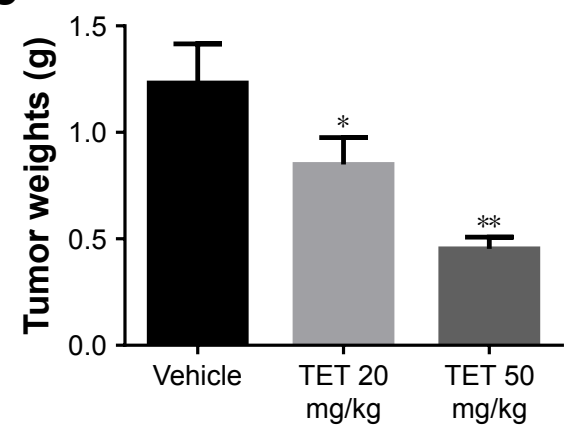

D

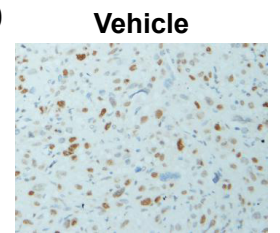

F

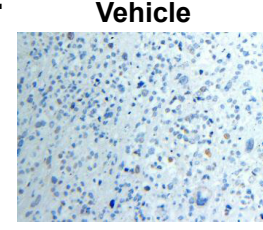

TET $20 \mathrm{mg} / \mathrm{kg}$
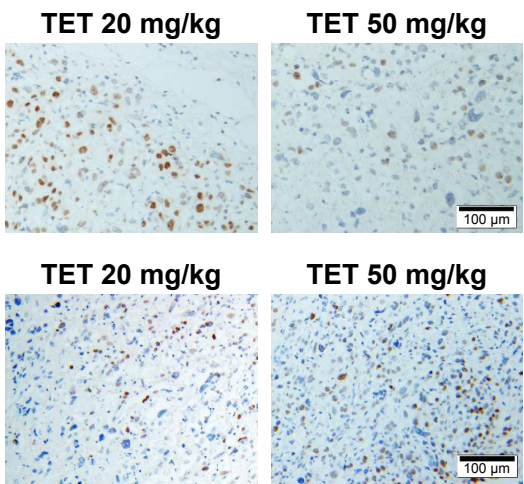

TET $50 \mathrm{mg} / \mathrm{kg}$

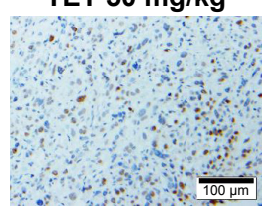

E

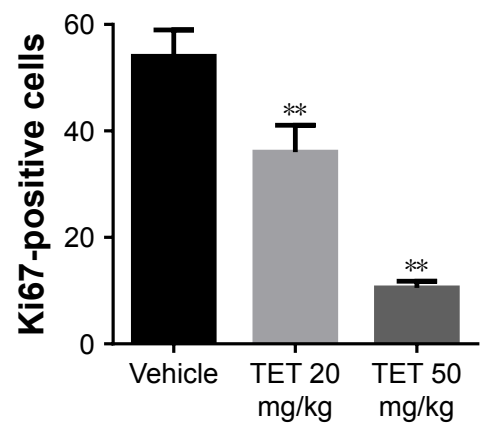

G

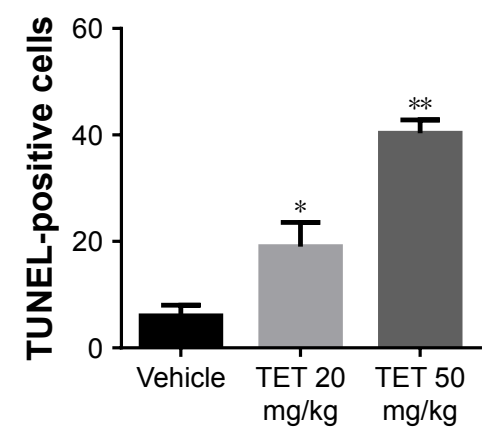

H

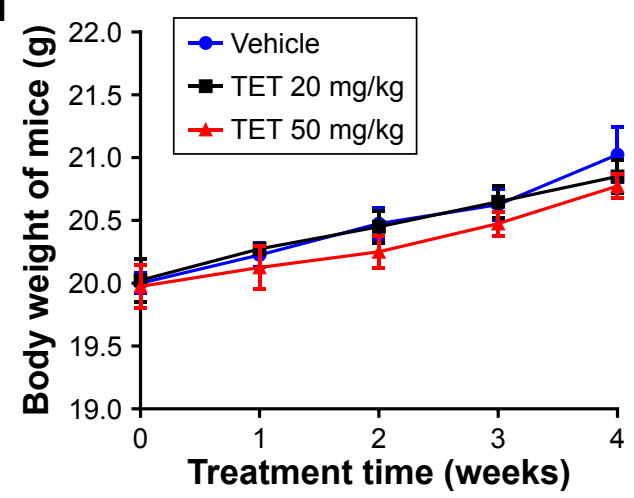

Figure 4 TET inhibited tumor growth by inducing apoptosis in vivo.

Notes: (A) The mice were subcutaneously inoculated with $5 \times 10^{6} \mathrm{SiHa}$ cells on the right flank. Mice were dosed with TET (0, 20, or $50 \mathrm{mg} / \mathrm{kg}$ ) for 4 weeks and the tumor volumes were monitored weekly. $* P<0.05, * * P<0.01$ vs vehicle group, $n=4$. (B) The mice were sacrificed after 4 weeks' treatment and the tumors were isolated and imaged. (C) Tumor weights were quantified in each group. $* P<0.05$, $* * P<0.01$ vs vehicle group, $n=4$. (D) Ki67 immunohistochemical staining of tumor tissues in each group. (E) Quantification of Ki67-positive cells in tumors. ${ }^{* *} P<0.01$ vs vehicle group, $n=4$. (F) TUNEL staining of tumor tissues in each group. (G) Quantification of Ki67-positive cells in tumors. $* P<0.05,{ }^{*} * P<0.01$ vs vehicle group, $n=4$. $(\mathbf{H})$ The body weight changes of mice treated with TET $(0,20$, or $50 \mathrm{mg} / \mathrm{kg})$ for 4 weeks, $\mathrm{n}=4$.

Abbreviation: TET, tetrandrine.

TET (Figure 4D and E). Moreover, the data of TUNEL indicated that either 20 or $50 \mathrm{mg} / \mathrm{kg}$ of TET resulted in prominent apoptosis in tumor tissues compared with the vehicle group (Figure 4F and G). Besides, there was no significant body weight changes of mice between vehicle and TET-treated group (Figure 4H). Thereby, we deduced that in the present study 20 or $50 \mathrm{mg} / \mathrm{kg}$ TET had no obvious adverse effect on mice. All these data demonstrated that TET suppressed cervical tumor growth in vivo by inducing apoptosis, which was in accord with the phenomenon in vitro.

\section{TET upregulated active caspase 3 and downregulated MMP2 and MMP9 expressions in cervical tumors}

To clarify the molecular mechanisms underlying the antitumor effect of TET on cervical cancer in vivo, we mainly focused on the expressions of active caspase 3, MMP2, and MMP9. As shown in Figure 5A-D, the level of active caspase 3 was notably increased, while invasion-associated proteins MMP2 and MMP9 were markedly decreased in TET-treated groups. All these data confirmed that TET 
A

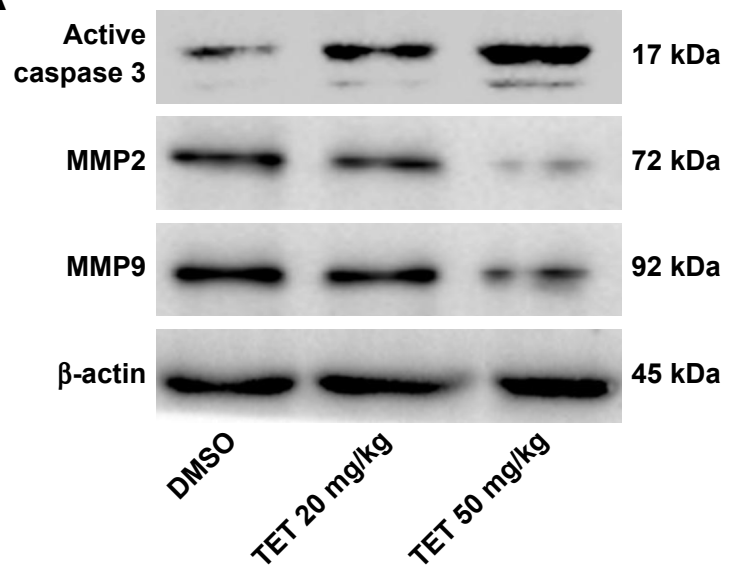

C

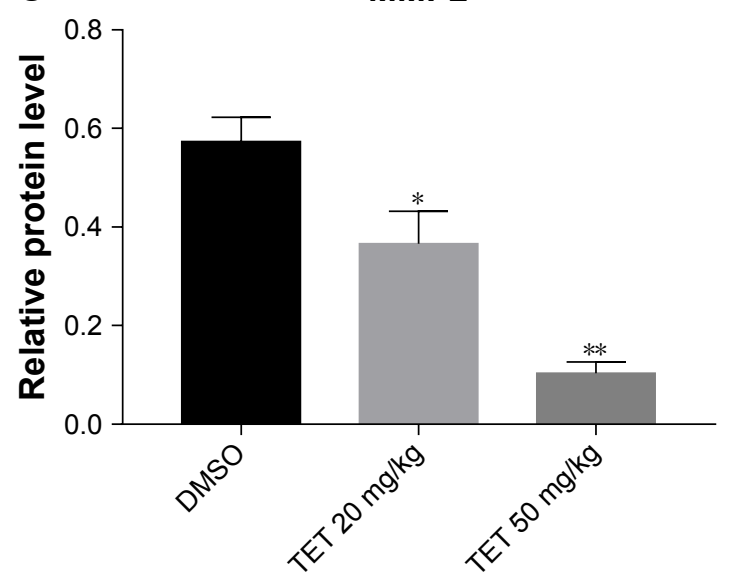

B

Active caspase 3

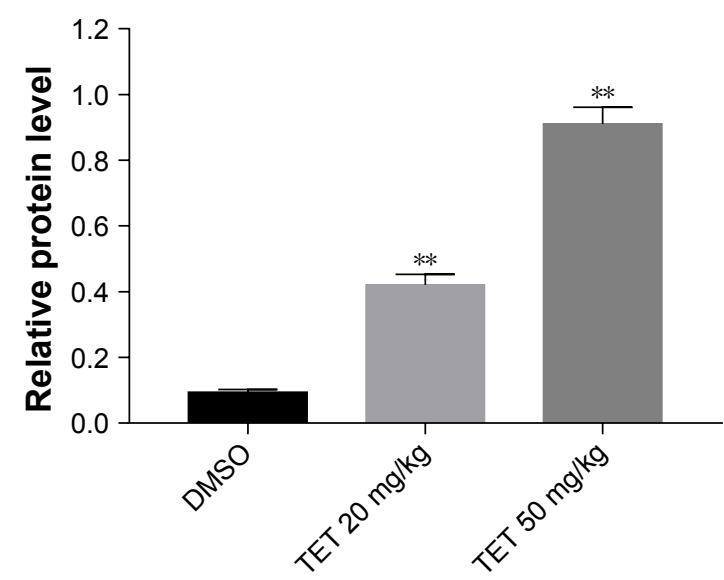

D

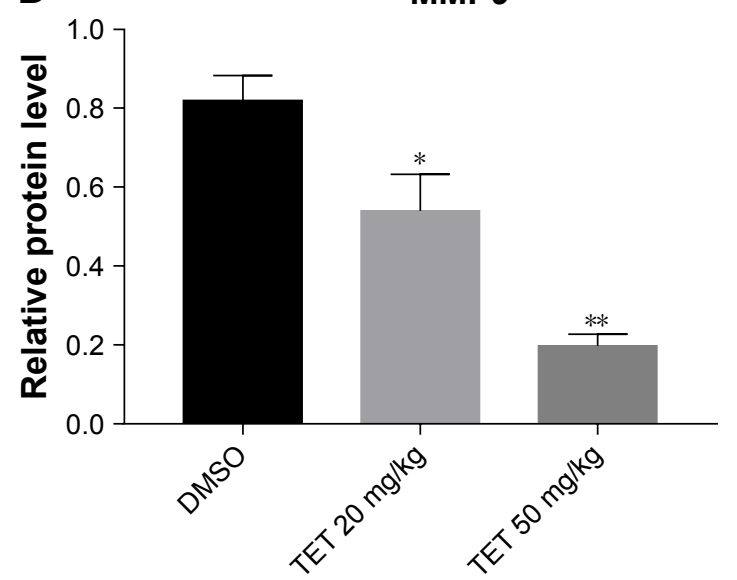

Figure 5 TET upregulated active caspase 3 level and suppressed MMP2 and MMP9 expressions in vivo.

Notes: (A) Relative protein levels of active caspase 3, MMP2, and MMP9 in mice treated with TET (0, 20, or $50 \mathrm{mg} / \mathrm{kg})$ for 4 weeks were detected by Western blot assay. (B) The relative expressions of active caspase 3 in tumors were quantified by normalizing to $\beta$-actin. $* * P<0.01$ vs vehicle group, $n=4$. (C) The relative expressions of MMP 2 in tumors were quantified by normalizing to $\beta$-actin. $* P<0.05, * * P<0.01$ vs vehicle group, $n=4$. (D) The relative expressions of MMP9 in tumors were quantified by normalizing to $\beta$-actin. $* P<0.05, * * P<0.01$ vs vehicle group, $n=4$.

Abbreviations: DMSO, dimethyl sulfoxide; MMP, matrix metalloproteinases; TET, tetrandrine.

significantly inhibited tumor growth, migration, and invasion in cervical cancer by upregulating active caspase 3 and downregulating MMP2 and MMP9 in vitro and in vivo, respectively.

\section{Discussion}

Accumulating evidence have indicated that TET is capable of exerting significant antitumor activities against various cancer cells in vitro and in vivo. ${ }^{17}$ However, the effects of TET on human cervical cancer have not been reported. Thus, in the present study, SiHa cells were used to examine the therapeutic effects of TET on cervical cancer in vitro and in vivo. In this study, we provide convincing evidence that TET significantly inhibited tumor growth, migration, and invasion in cervical cancer by inducing apoptosis in vitro and in vivo; these events were associated with the activation of apoptosis-associated protein casaspe 3 and suppression of migration-associated proteins. To the best of our knowledge, this is the first report of the effects of TET on human cervical cancer.

Cervical cancer is the most common malignancy of uterus in women. ${ }^{1}$ The results of the present study revealed that TET was capable of inhibiting cervical cancer cell proliferation in a dose- and time-dependent manners. These results are consistent with previously reported study that TET showed a concentration- and time-dependent inhibition of colon cancer cells proliferation. ${ }^{14}$ Our results indicated that $\mathrm{SiHa}$ cells were sensitive to TET to a degree that was similar with the cell types reported in this literature. Since TET showed significant cytotoxic effects on $\mathrm{SiHa}$ cells, we next investigated its antitumor effects in vivo. The in vivo data showed that TET markedly inhibited the growth of subcutaneous 
tumors; higher dose $(50 \mathrm{mg} / \mathrm{kg})$ was more potent than lower dose $(20 \mathrm{mg} / \mathrm{kg})$. Our finding was consistent with that of Wu et al, who demonstrated that TET ( $50 \mathrm{mg} / \mathrm{kg})$ effectively suppressed tumor growth in colon cancer. ${ }^{14}$ All these results suggested that TET exerted notable antitumor effects on cervical cancer in vitro and in vivo.

Apoptosis plays an important role in killing tumor cells. ${ }^{18} \mathrm{Li}$ et al found that TET could induce human colon cancer cells apoptosis by activating the levels of caspase $3 .{ }^{19}$ Zhang et al also revealed that a combination of TET with cisplatin significantly enhanced cytotoxicity in ovarian cancer by inducing apoptosis in vitro and in vivo. ${ }^{20}$ In addition, Chang et al found that TET $(10 \mathrm{mg} / \mathrm{kg})$ markedly inhibited pulmonary metastases in CT26 colorectal adenocarcinoma-bearing BALB/c mice. ${ }^{21}$ Similarly, our results revealed that TET significantly upregulated proapoptotic protein active caspase 3 in SiHa cells. Moreover, in vivo data further demonstrated that TET could inhibit tumor growth by upregulating active caspase 3. All these results confirmed that TET inhibited cervical cancer growth, migration, and invasion in vitro and in vivo.

Most malignant tumors exhibit a highly migratory and invasive capacity, which is closely associated with tumor metastasis. ${ }^{22}$ Liu et al found that TET inhibited the migration and invasion in human prostate cancer cells. Therefore, wound healing assay and transwell assay were used to evaluate the effects of TET on the migration and invasion of SiHa cells, respectively. We found that TET exerted a significant inhibitory effects on the migration and invasion of SiHa cells. Thus, the molecular mechanisms by which TET regulated the migration and invasion of cervical cancer cells were investigated. MMPs are the main proteases for invasion and degradation of basement membranes and extracellular matrix. ${ }^{23}$ MMP2 and MMP9 are capable of cleaving gelatine and provide favorable conditions for cell migration and invasion. ${ }^{23}$ TET has been identified to reduce the invasiveness and migration of human colorectal adenocarcinoma cells in vitro and in vivo by inhibiting the expressions of MMP2 and MMP9. ${ }^{24}$ Moreover, Qi et al revealed that TET inhibited migration and invasion of rheumatoid arthritis fibroblast cells by downregulating the levels of MMP2 and MMP9. ${ }^{23}$ Our finding is consistent with these reports and confirmed that TET suppressed the migration and invasion by inhibiting MMP2 and MMP9 in cervical cancer cells in vitro and in vivo.

\section{Conclusion}

In summary, this study demonstrates that TET inhibited tumor growth, migration, and invasion in cervical cancer in vitro and in vivo. Therefore, these findings provide a strong basis for further exploration of TET as a therapeutic agent for the treatment of cervical cancer.

\section{Disclosure}

The authors report no conflicts of interest in this work.

\section{References}

1. Hu Z, Yu L, Zhu D, et al. Disruption of HPV16-E7 by CRISPR/Cas system induces apoptosis and growth inhibition in HPV16 positive human cervical cancer cells. Biomed Res Int. 2014;2014:612823-9.

2. Manzo-Merino J, Contreras-Paredes A, Vázquez-Ulloa E, RochaZavaleta L, Fuentes-Gonzalez AM, Lizano M. The role of signaling pathways in cervical cancer and molecular therapeutic targets. Arch Med Res. 2014;45(7):525-539.

3. Clifford GM, Smith JS, Plummer M, Muñoz N, Franceschi S. Human papillomavirus types in invasive cervical cancer worldwide: a metaanalysis. Br J Cancer. 2003;88(1):63-73.

4. Muñoz N, Bosch FX, de Sanjosé S. International Agency for Research on Cancer Multicenter Cervical Cancer Study Group. Epidemiologic classification of human papillomavirus types associated with cervical cancer. N Engl J Med. 2003;348(6):518-527.

5. Zhao Y, Moran MS, Yang Q, et al. Metadherin regulates radioresistance in cervical cancer cells. Oncol Rep. 2012;27(5):1520-1526.

6. Chemoradiotherapy for Cervical Cancer Meta-Analysis Collaboration. Reducing uncertainties about the effects of chemoradiotherapy for cervical cancer: a systematic review and meta-analysis of individual patient data from 18 randomized trials. J Clin Oncol. 2008;26(35): 5802-5812.

7. Shi C, Thum C, Zhang Q, et al. Inhibition of the cancer-associated TASK 3 channels by magnetically induced thermal release of Tetrandrine from a polymeric drug carrier. J Control Release. 2016;237:50-60.

8. Liu W, Kou B, Ma ZK, et al. Tetrandrine suppresses proliferation, induces apoptosis, and inhibits migration and invasion in human prostate cancer cells. Asian J Androl. 2015;17(5):850-853.

9. He FQ, Qiu BY, Li TK, et al. Tetrandrine suppresses amyloid- $\beta$-induced inflammatory cytokines by inhibiting NF- $\mathrm{B}$ pathway in murine BV2 microglial cells. Int Immunopharmacol. 2011;11(9):1220-1225.

10. Huang $\mathrm{P}, \mathrm{Xu} \mathrm{Y}$, Wei R, et al. Efficacy of tetrandrine on lowering intraocular pressure in animal model with ocular hypertension. J Glaucoma. 2011;20(3):183-188.

11. Li X, Lu X, Xu H, et al. Paclitaxel/tetrandrine coloaded nanoparticles effectively promote the apoptosis of gastric cancer cells based on "oxidation therapy". Mol Pharm. 2012;9(2):222-229.

12. Liu C, Gong K, Mao X, Li W. Tetrandrine induces apoptosis by activating reactive oxygen species and repressing Akt activity in human hepatocellular carcinoma. Int J Cancer. 2011;129(6):1519-1531.

13. Shi C, Ahmad Khan S, Wang K, Schneider M. Improved delivery of the natural anticancer drug tetrandrine. Int J Pharm. 2015;479(1):41-51.

14. Wu JM, Chen Y, Chen JC, Lin TY, Tseng SH. Tetrandrine induces apoptosis and growth suppression of colon cancer cells in mice. Cancer Lett. 2010;287(2):187-195.

15. Chen Y, Chen JC, Tseng SH. Tetrandrine suppresses tumor growth and angiogenesis of gliomas in rats. Int $J$ Cancer. 2009;124(10): 2260-2269.

16. Amann A, Zwierzina M, Gamerith G, et al. Development of an innovative $3 \mathrm{D}$ cell culture system to study tumour - stroma interactions in non-small cell lung cancer cells. PLoS One. 2014;9(3):e92511.

17. Chen $\mathrm{T}, \mathrm{Ji} \mathrm{B}, \mathrm{Chen} Y$. Tetrandrine triggers apoptosis and cell cycle arrest in human renal cell carcinoma cells. J Nat Med. 2014;68(1):46-52.

18. Choudhari AS, Suryavanshi SA, Kaul-Ghanekar R. The aqueous extract of Ficus religiosa induces cell cycle arrest in human cervical cancer cell lines $\mathrm{SiHa}$ (HPV-16 Positive) and apoptosis in HeLa (HPV-18 positive). PLoS One. 2013;8(7):e70127. 
19. Li X, Zhen D, Lu X, et al. Enhanced cytotoxicity and activation of ROSdependent c-Jun NH2-terminal kinase and caspase-3 by low doses of tetrandrine-loaded nanoparticles in Lovo cells - a possible Trojan strategy against cancer. Eur J Pharm Biopharm. 2010;75(3):334-340.

20. Zhang Y, Wang C, Wang H, Wang K, du Y, Zhang J. Combination of Tetrandrine with cisplatin enhances cytotoxicity through growth suppression and apoptosis in ovarian cancer in vitro and in vivo. Cancer Lett. 2011;304(1):21-32.

21. Chang $\mathrm{KH}$, Liao HF, Chang $\mathrm{HH}$, et al. Inhibitory effect of tetrandrine on pulmonary metastases in CT26 colorectal adenocarcinoma-bearing BALB/c mice. Am J Chin Med. 2004;32(6):863-872.
22. Ma X, Miao H, Jing B, et al. Claudin- 4 controls the proliferation, apoptosis, migration and in vivo growth of MCF-7 breast cancer cells. Oncol Rep. 2015;34(2):681-690.

23. Zhang XH, Xu XX, Xu T. Ginsenoside Ro suppresses interleukin-1ßinduced apoptosis and inflammation in rat chondrocytes by inhibiting NF-кB. Chin J Nat Med. 2015;13(4):283-289.

24. Horng CT, Yang JS, Chiang JH, et al. Inhibitory effects of tetrandrine on epidermal growth factor-induced invasion and migration in HT29 human colorectal adenocarcinoma cells. Mol Med Rep. 2016;13(1): $1003-1009$

\section{Publish your work in this journal}

Drug Design, Development and Therapy is an international, peerreviewed open-access journal that spans the spectrum of drug design and development through to clinical applications. Clinical outcomes, patient safety, and programs for the development and effective, safe, and sustained use of medicines are the features of the journal, which has also been accepted for indexing on PubMed Central. The manuscript management system is completely online and includes a very quick and fair peer-review system, which is all easy to use. Visit http://www.dovepress.com/testimonials.php to read real quotes from published authors.

Submit your manuscript here: http://www.dovepress.com/drug-design-development-and-therapy-journal 